Research Article

\title{
Observer Design for a Class of Nonlinear Descriptor Systems: A Takagi-Sugeno Approach with Unmeasurable Premise Variables
}

\author{
J. Soulami, ${ }^{1}$ A. El Assoudi, ${ }^{1}$ M. Essabre, ${ }^{1}$ M. Habibi, ${ }^{2}$ and E. El Yaagoubi ${ }^{1}$ \\ ${ }^{1}$ ECPI, Department of Electrical Engineering, ENSEM, University Hassan II of Casablanca, BP 8118, Oasis, 20103 Casablanca, Morocco \\ ${ }^{2}$ ESPCM, Department of Physics, Faculty of Science, University Ibn Tofail of Kenitra, BP 133, 14000 Kenitra, Morocco
}

Correspondence should be addressed to J. Soulami; jalal.soulami@gmail.com

Received 18 June 2014; Revised 20 January 2015; Accepted 8 March 2015

Academic Editor: Ai-Guo Wu

Copyright (C) 2015 J. Soulami et al. This is an open access article distributed under the Creative Commons Attribution License, which permits unrestricted use, distribution, and reproduction in any medium, provided the original work is properly cited.

\begin{abstract}
The Takagi-Sugeno (T-S) fuzzy observer for dynamical systems described by ordinary differential equations is widely discussed in the literature. The aim of this paper is to extend this observer design to a class of T-S descriptor systems with unmeasurable premise variables. In practice, the computation of solutions of differential-algebraic equations requires the combination of an ordinary differential equations (ODE) routine together with an optimization algorithm. Therefore, a natural way permitting to estimate the state of such a system is to design a procedure based on a similar numerical algorithm. Beside some numerical difficulties, the drawback of such a method lies in the fact that it is not easy to establish a rigorous proof of the convergence of the observer. The main result of this paper consists in showing that the state estimation problem for a class of T-S descriptor systems can be achieved by using a fuzzy observer having only an ODE structure. The convergence of the state estimation error is studied using the Lyapunov theory and the stability conditions are given in terms of linear matrix inequalities (LMIs). Finally, an application to a model of a heat exchanger pilot process is given to illustrate the performance of the proposed observer.
\end{abstract}

\section{Introduction}

In practice, the control and the supervision of a process require the knowledge of the state of the process. One way permitting to obtain such unknown state consists in using physical sensors. However, in many cases and due to a high running cost and physical constraints, this method becomes very limited. To solve this problem, one solution is to design an observer. This method combines a priori knowledge about a physical system (nominal model) with experimental data (some on-line measurements) to provide on-line estimation of states and/or parameters. In the present work, we are concerned with the problem of the observer synthesis for nonlinear descriptor systems that can be described by dynamic models of T-S descriptor with unmeasurable premise variables. In fact, many chemical and physical processes can be described by nonlinear systems of differential and algebraic equations [1-3]. These systems are variously called descriptor systems, singular systems, or differential algebraic equations (DAEs). This formulation includes both dynamic and static relations. Consequently, this formalism is much more general than the usual one and can model the physical constraints or impulsive behavior due to an improper part of the system. The numerical simulation of such descriptor models usually combines an ODE numerical method together with an optimization algorithm.

Recently, there has been a great deal of interest in using the approach based on the representation of the nonlinear systems by T-S models [4]. This interest relies on the fact that once the T-S fuzzy models are obtained, some analysis and design tools developed in the linear system can be used, which facilitates observer or/and controller synthesis for complex nonlinear systems. Many practical problems using T-S fuzzy approach have been widely treated in the literature. The stability and stabilization problems of T-S fuzzy systems can be found in [5-7]. In [8] a filter for nonuniformly sampled nonlinear systems represented by T-S model is proposed. For filtering problem in networked 
control systems where the nonlinear discrete-time system is modeled by T-S fuzzy model, we can cite [9]. The T-S fuzzy observer problems for dynamic T-S fuzzy models described by ordinary differential equations (ODEs) with measurable and unmeasurable premise variables are studied in [10-20]. Concerning nonlinear descriptor systems described by T-S descriptor models the problem of fuzzy observer design has been widely investigated; see, for instance, [21-25]. The aim of this paper is to give a fuzzy observer design to a class of fuzzy descriptor systems permitting to estimate the unknown state without the use of an optimization algorithm. The idea of the proposed result is to separate the dynamic relations of the static relations in the descriptor model.

The outline of the paper is as follows. The main result is stated in Sections 2 and 3. It consists in showing that the state estimation problem for a class of fuzzy descriptor systems can be achieved by using a fuzzy observer having only an ODE structure. First, the method used for decomposing the differential part of the algebraic part is developed; secondly we give a fuzzy observer design permitting to estimate the unknown state. In Section 4, we illustrate the performance of the proposed observer in simulation through a model of a heat exchanger pilot process.

\section{Fuzzy Descriptor Systems}

The form of the class of Takagi-Sugeno descriptor systems with unmeasurable premise variables studied in this paper is

$$
\begin{gathered}
E \dot{x}=\sum_{i=1}^{q} h_{i}(x)\left(A_{i} x+B_{i} u\right), \\
y=C x,
\end{gathered}
$$

where $x=\left(\begin{array}{ll}X_{1}^{T} & X_{2}^{T}\end{array}\right)^{T} \in \mathbf{R}^{n}$ is the state vector with $X_{1} \in \mathbf{R}^{r}$, $X_{2} \in \mathbf{R}^{n-r}, u \in \mathbf{R}^{m}$ is the control input, and $y \in \mathbf{R}^{p}$ is the measured output. $E \in \mathbf{R}^{n \times n}$ with $\operatorname{rank}(E)=r, A_{i} \in \mathbf{R}^{n \times n}$, $B_{i} \in \mathbf{R}^{n \times m}$, and $C \in \mathbf{R}^{p \times n}$ are real known constant matrices with

$$
\begin{gathered}
E=\left(\begin{array}{ll}
I & 0 \\
0 & 0
\end{array}\right), \quad A_{i}=\left(\begin{array}{ll}
A_{11 i} & A_{12 i} \\
A_{21 i} & A_{22 i}
\end{array}\right), \\
B_{i}=\left(\begin{array}{l}
B_{1 i} \\
B_{2 i}
\end{array}\right), \quad C=\left(\begin{array}{ll}
C_{1} & C_{2}
\end{array}\right),
\end{gathered}
$$

where $A_{22 i}$ constant matrices are invertible $\left(\operatorname{rank}\left(A_{22 i}\right)=n-\right.$ $r) . q$ is the number of submodels. The $h_{i}(x)$ are the weighting functions that ensure the transition between the contribution of each submodel:

$$
\begin{gathered}
E \dot{x}=A_{i} x+B_{i} u, \\
y=C x .
\end{gathered}
$$

They depend on unmeasurable premise variables (state of the system) and have the following properties:

$$
\begin{gathered}
\sum_{i=1}^{q} h_{i}(x)=1, \\
0 \leq h_{i}(x) \leq 1, \quad i=1, \ldots, q .
\end{gathered}
$$

In order to design an observer for each submodel (3) $(i=$ $1, \ldots, q)$, we will make the following assumptions.

(H1) $\left(E, A_{i}\right)$ is regular; that is, $\operatorname{det}\left(s E-A_{i}\right) \neq 0 \forall s \in \mathbf{C}$.

(H2) All submodels (3) are impulse observable; that is,

$$
\operatorname{rank}\left(\left(\begin{array}{cc}
E & A_{i} \\
0 & E \\
0 & C
\end{array}\right)\right)=n+\operatorname{rank}(E)
$$

(H3) All submodels (3) are detectable; that is,

$$
\operatorname{rank}\left(\left(\begin{array}{c}
s E-A_{i} \\
C
\end{array}\right)\right)=n \quad \forall s \in \mathbf{C} .
$$

To design a fuzzy observer for system (1), our approach is based on the separate dynamic relations of the static relations for each submodel (3) and the global model is obtained by aggregation of the submodels.

Thus, from (2), system (3) can be written as follows:

$$
\begin{gathered}
\dot{X}_{1}=A_{11 i} X_{1}+A_{12 i} X_{2}+B_{1 i} u, \\
0=A_{21 i} X_{1}+A_{22 i} X_{2}+B_{2 i} u, \\
y=C_{1} X_{1}+C_{2} X_{2} .
\end{gathered}
$$

Using the fact that $A_{22 i}^{-1}$ exist, system (7) can be rewritten as

$$
\begin{gathered}
\dot{X}_{1}=M_{i} X_{1}+N_{i} u, \\
X_{2}=Q_{i} X_{1}+R_{i} u, \\
y=S_{i} X_{1}+G_{i} u,
\end{gathered}
$$

where

$$
\begin{aligned}
M_{i} & =A_{11 i}-A_{12 i} A_{22 i}^{-1} A_{21 i}, \\
N_{i} & =B_{1 i}-A_{12 i} A_{22 i}^{-1} B_{2 i}, \\
Q_{i} & =-A_{22 i}^{-1} A_{21 i}, \\
R_{i} & =-A_{22 i}^{-1} B_{2 i}, \\
S_{i} & =\left(C_{1}-C_{2} A_{22 i}^{-1} A_{21 i}\right), \\
G_{i} & =-C_{2} A_{22 i}^{-1} B_{2 i} .
\end{aligned}
$$


Then, the fuzzy descriptor system (1) can be rewritten in the following form:

$$
\begin{gathered}
\dot{X}_{1}=\sum_{i=1}^{q} \bar{h}_{i}(x)\left(M_{i} X_{1}+N_{i} u\right), \\
X_{2}=\sum_{i=1}^{q} \bar{h}_{i}(x)\left(Q_{i} X_{1}+R_{i} u\right), \\
y=\sum_{i=1}^{q} \bar{h}_{i}(x)\left(S_{i} X_{1}+G_{i} u\right),
\end{gathered}
$$

where

$$
\bar{h}_{i}(x)=h_{i}\left(X_{1}, X_{2}=Q_{i} X_{1}+R_{i} u\right)=\bar{h}_{i}\left(X_{1}, u\right) .
$$

\section{Fuzzy Observer Design}

In this section, our aim is to design a fuzzy observer for descriptor system (1). Based on the separate dynamic relations of the static relations in the descriptor model (1) (see (10)) and by substituting (11) in (10), the proposed observer is given by the following equations:

$$
\begin{aligned}
\dot{\bar{X}}_{1} & =\sum_{i=1}^{q} \bar{h}_{i}\left(\widehat{X}_{1}, u\right)\left(M_{i} \widehat{X}_{1}+N_{i} u-L_{i}(\hat{y}-y)\right), \\
\widehat{X}_{2} & =\sum_{i=1}^{q} \bar{h}_{i}\left(\widehat{X}_{1}, u\right)\left(Q_{i} \widehat{X}_{1}+R_{i} u\right), \\
\hat{y} & =\sum_{i=1}^{q} \bar{h}_{i}\left(\widehat{X}_{1}, u\right)\left(S_{i} \widehat{X}_{1}+G_{i} u\right),
\end{aligned}
$$

where $\widehat{X}_{1}, \widehat{X}_{2}$, and $\hat{y}$ denote the estimated state vectors of $X_{1}$, $X_{2}$, and output vector $y$, respectively. The local gains $L_{i}$ can be determined by Theorem 1 .

In order to establish the conditions for the asymptotic convergence of the observer (12), we define the state estimation error:

$$
e=\left(\begin{array}{l}
e_{1} \\
e_{2}
\end{array}\right)=\left(\begin{array}{l}
\widehat{X}_{1}-X_{1} \\
\widehat{X}_{2}-X_{2}
\end{array}\right) .
$$

It follows from (10) and (12) that the observer error dynamic is given by the differential and algebraic equations:

$$
\begin{aligned}
\dot{e}_{1}= & \sum_{i=1}^{q} \bar{h}_{i}\left(\widehat{X}_{1}, u\right)\left(M_{i} \widehat{X}_{1}+N_{i} u-L_{i}(\widehat{y}-y)\right) \\
& -\sum_{i=1}^{q} \bar{h}_{i}\left(X_{1}, u\right)\left(M_{i} X_{1}+N_{i} u\right) \\
e_{2}= & \sum_{i=1}^{q} \bar{h}_{i}\left(\widehat{X}_{1}, u\right)\left(Q_{i} \widehat{X}_{1}+R_{i} u\right) \\
& -\sum_{i=1}^{q} \bar{h}_{i}\left(X_{1}, u\right)\left(Q_{i} X_{1}+R_{i} u\right)
\end{aligned}
$$

By adding and subtracting the term $\sum_{i=1}^{q} \bar{h}_{i}\left(\widehat{X}_{1}, u\right)\left(M_{i} X_{1}+\right.$ $N_{i} u$ ), (14) becomes

$$
\begin{aligned}
\dot{e}_{1}= & \sum_{i=1}^{q} \bar{h}_{i}\left(\widehat{X}_{1}, u\right)\left(M_{i} e_{1}-L_{i}(\hat{y}-y)\right) \\
& -\sum_{i=1}^{q}\left(\bar{h}_{i}\left(X_{1}, u\right)-\bar{h}_{i}\left(\widehat{X}_{1}, u\right)\right)\left(M_{i} X_{1}+N_{i} u\right) .
\end{aligned}
$$

Note that

$$
\begin{aligned}
& \sum_{i=1}^{q}\left(\bar{h}_{i}\left(X_{1}, u\right)-\bar{h}_{i}\left(\widehat{X}_{1}, u\right)\right) M_{i} \\
& =\sum_{i, j=1}^{q} \bar{h}_{i}\left(X_{1}, u\right) \bar{h}_{j}\left(\widehat{X}_{1}, u\right)\left(M_{i}-M_{j}\right), \\
& \sum_{i=1}^{q}\left(\bar{h}_{i}\left(X_{1}, u\right)-\bar{h}_{i}\left(\widehat{X}_{1}, u\right)\right) N_{i} \\
& =\sum_{i, j=1}^{q} \bar{h}_{i}\left(X_{1}, u\right) \bar{h}_{j}\left(\widehat{X}_{1}, u\right)\left(N_{i}-N_{j}\right) .
\end{aligned}
$$

Then, (16) becomes

$$
\begin{aligned}
\dot{e}_{1}= & \sum_{i=1}^{q} \bar{h}_{i}\left(\widehat{X}_{1}, u\right)\left(M_{i} e_{1}-L_{i}(\hat{y}-y)\right) \\
& -\sum_{i, j=1}^{q}\left(\bar{h}_{i}\left(X_{1}, u\right) \bar{h}_{j}\left(\widehat{X}_{1}, u\right)\right)\left(\Delta M_{i j} X_{1}+\Delta N_{i j} u\right),
\end{aligned}
$$

where $\Delta M_{i j}=M_{i}-M_{j}$ and $\Delta N_{i j}=N_{i}-N_{j}$.

Similarly $y$ can be written as follows:

$$
\begin{aligned}
y=\sum_{i, k=1}^{q} \bar{h}_{i}\left(X_{1}, u\right) \bar{h}_{k}\left(\widehat{X}_{1}, u\right) \\
\cdot\left(\left(S_{k}+\Delta S_{i k}\right) X_{1}+\left(G_{k}+\Delta G_{i k}\right) u\right),
\end{aligned}
$$

where $\Delta S_{i k}=S_{i}-S_{k}$ and $\Delta G_{i k}=G_{i}-G_{k}$.

Multiplying by $\sum_{i=1}^{q} \bar{h}_{i}\left(X_{1}, u\right)$, we obtain

$$
\begin{aligned}
\dot{e}_{1}= & \sum_{i, j=1}^{q} \bar{h}_{i}\left(X_{1}, u\right) \bar{h}_{j}\left(\widehat{X}_{1}, u\right)\left(M_{j} e_{1}-L_{j}(\widehat{y}-y)\right) \\
& -\sum_{i, j=1}^{q}\left(\bar{h}_{i}\left(X_{1}, u\right) \bar{h}_{j}\left(\widehat{X}_{1}, u\right)\right)\left(\Delta M_{i j} X_{1}+\Delta N_{i j} u\right),
\end{aligned}
$$

$$
\widehat{y}=\sum_{i, k=1}^{q} \bar{h}_{i}\left(X_{1}, u\right) \bar{h}_{k}\left(\widehat{X}_{1}, u\right)\left(S_{k} \widehat{X}_{1}+G_{k} u\right) .
$$

By substituting (19) and (21) in (20), we obtain

$$
\begin{gathered}
\dot{e}_{1}=\sum_{i, j, k=1}^{q} \bar{h}_{i}\left(X_{1}, u\right) \bar{h}_{j}\left(\widehat{X}_{1}, u\right) \bar{h}_{k}\left(\widehat{X}_{1}, u\right) \\
\cdot\left(\Omega_{j k} e_{1}+\Gamma_{i j k} X_{1}+\Lambda_{i j k} u\right),
\end{gathered}
$$


where

$$
\begin{aligned}
& \Omega_{j k}=M_{j}-L_{j} S_{k}, \\
& \Gamma_{i j k}=L_{j}\left(S_{i}-S_{k}\right)-\left(M_{i}-M_{j}\right), \\
& \Lambda_{i j k}=L_{j}\left(G_{i}-G_{k}\right)-\left(N_{i}-N_{j}\right), \\
& \quad i, j, k \in\{1, \ldots, q\} .
\end{aligned}
$$

By adding and subtracting the term $\sum_{i=1}^{q} \bar{h}_{i}\left(\widehat{X}_{1}, u\right)\left(Q_{i} X_{1}+\right.$ $R_{i} u$ ), (15) becomes

$$
\begin{aligned}
e_{2}= & \sum_{i=1}^{q} \bar{h}_{i}\left(\widehat{X}_{1}, u\right) Q_{i} e_{1} \\
& -\sum_{i=1}^{q}\left(\bar{h}_{i}\left(X_{1}, u\right)-\bar{h}_{i}\left(\widehat{X}_{1}, u\right)\right)\left(Q_{i} X_{1}+R_{i} u\right) .
\end{aligned}
$$

Note that to prove the convergence of the estimation error $e$ toward zero, it suffices to prove that $e_{1}$ converges to zero. Thus, let $\widetilde{X}_{1}=\left(\begin{array}{ll}e_{1}^{T} & X_{1}^{T}\end{array}\right)^{T}$; we have

$$
\begin{gathered}
\dot{\bar{X}}_{1}=\sum_{i, j, k=1}^{q} \bar{h}_{i}\left(X_{1}, u\right) \bar{h}_{j}\left(\widehat{X}_{1}, u\right) \\
\cdot \bar{h}_{k}\left(\widehat{X}_{1}, u\right)\left(\mathscr{H}_{i j k} \widetilde{X}_{1}+\mathscr{T}_{i j k} u\right), \\
z_{1}=H \widetilde{X}_{1},
\end{gathered}
$$

where

$$
\begin{gathered}
\mathscr{H}_{i j k}=\left(\begin{array}{cc}
\Omega_{j k} & \Gamma_{i j k} \\
0 & M_{i}
\end{array}\right), \\
\mathscr{T}_{i j k}=\left(\begin{array}{c}
\Lambda_{i j k} \\
N_{i}
\end{array}\right), \\
H=\left(\begin{array}{ll}
I & 0
\end{array}\right) .
\end{gathered}
$$

Thus, the aim is to determine the observer gains $L_{i}(i=$ $1, \ldots, q)$ to ensure the stability of (25) while attenuating the effect of the input $u$ on $z_{1}$. Therefore, the convergence condition of the observer (12) can be formulated by the following theorem.

Theorem 1. Under the above hypotheses (H1), (H2), and (H3), the state error between the T-S descriptor model (1) and its observer (12) converges asymptotically towards zero, if there exist symmetric positive definite matrices $P_{1}$ and $P_{2}$, matrices $\mathscr{K}_{i}, i=1, \ldots, q$, and a positive scalar $\beta$, such that the following LMIs hold:

$$
\left(\begin{array}{ccc}
\mathscr{Z}_{1 j k} & \Theta_{i j k} & \Psi_{i j k} \\
\Theta_{i j k}^{T} & \mathscr{Z}_{2 i} & P_{2} N_{i} \\
\Psi_{i j k}^{T} & N_{i}^{T} P_{2} & -\beta I
\end{array}\right)<0 \quad \forall(i, j, k) \in\{1, \ldots, q\}^{3},
$$

where

$$
\begin{aligned}
\mathscr{Z}_{1 j k} & =M_{j}^{T} P_{1}+P_{1} M_{j}-\mathscr{K}_{j} S_{k}-S_{k}^{T} \mathscr{K}_{j}^{T}+I, \\
\mathscr{Z}_{2 i} & =M_{i}^{T} P_{2}+P_{2} M_{i}, \\
\Theta_{i j k} & =\mathscr{K}_{j}\left(S_{i}-S_{k}\right)-P_{1}\left(M_{i}-M_{j}\right), \\
\Psi_{i j k} & =\mathscr{K}_{j}\left(G_{i}-G_{k}\right)-P_{1}\left(N_{i}-N_{j}\right) .
\end{aligned}
$$

The gains of the observer are derived from

$$
L_{j}=P_{1}^{-1} \mathscr{K}_{j}
$$

and the attenuation level is

$$
\alpha=\sqrt{\beta} .
$$

Proof of Theorem 1. Consider the following quadratic Lyapunov function:

$$
V=\widetilde{X}_{1}^{T} P \widetilde{X}_{1}, \quad P=P^{T}>0
$$

with

$$
P=\left(\begin{array}{cc}
P_{1} & 0 \\
0 & P_{2}
\end{array}\right) .
$$

The time derivative of $V$ along the trajectory of (25) is given by

$$
\begin{aligned}
\dot{V}=\sum_{i, j, k=1}^{q} \bar{h}_{i}\left(X_{1}, u\right) \bar{h}_{j}\left(\widehat{X}_{1}, u\right) \bar{h}_{k}\left(\widehat{X}_{1}, u\right) \\
\cdot\left(\widetilde{X}_{1}^{T}\left(\mathscr{H}_{i j k}^{T} P+P \mathscr{H}_{i j k}\right) \widetilde{X}_{1}\right. \\
\left.+\widetilde{X}_{1}^{T} P \mathscr{T}_{i j k} u+u^{T} \mathscr{T}_{i j k}^{T} P \widetilde{X}_{1}\right) .
\end{aligned}
$$

In order to ensure the stability of (25) and the boundedness of the transfer from $u$ to $z_{1}$,

$$
\frac{\left\|z_{1}\right\|_{2}}{\|u\|_{2}}<\alpha, \quad\|u\|_{2} \neq 0,
$$

we consider the following criterion:

$$
\dot{V}+z_{1}^{T} z_{1}-\alpha^{2} u^{T} u<0 .
$$

From (25) and (33), inequality (35) becomes

$$
\begin{aligned}
& \sum_{i, j, k=1}^{q} \bar{h}_{i}\left(X_{1}, u\right) \bar{h}_{j}\left(\widehat{X}_{1}, u\right) \bar{h}_{k}\left(\widehat{X}_{1}, u\right)\left(\widetilde{X}_{1}^{T} u^{T}\right) \Sigma_{i j k}\left(\begin{array}{c}
\widetilde{X}_{1} \\
u
\end{array}\right) \\
& \quad<0
\end{aligned}
$$

where

$$
\Sigma_{i j k}=\left(\begin{array}{cc}
\mathscr{H}_{i j k}^{T} P+P \mathscr{H}_{i j k}+H^{T} H & P \mathscr{T}_{i j k} \\
\mathscr{T}_{i j k}^{T} P & -\alpha^{2} I
\end{array}\right) .
$$

The inequality (36) is satisfied if

$$
\Sigma_{i j k}<0 \quad \forall i, j, k \in\{1, \ldots, q\} .
$$




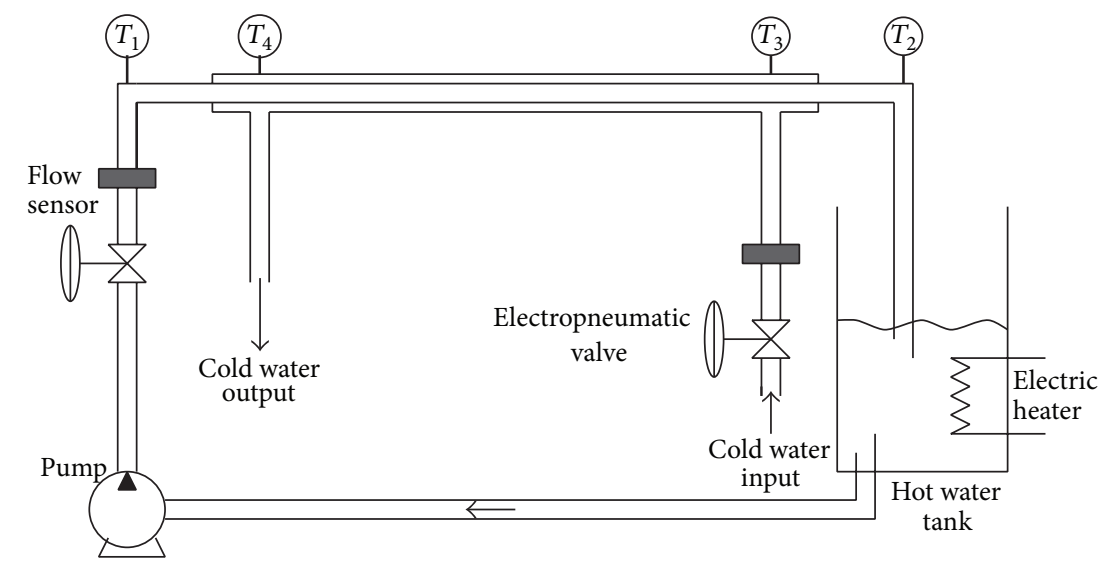

FIgURE 1: Heat exchanger plant.

Then, from (23), (26), and the use of the changes of variables,

$$
\begin{gathered}
\mathscr{K}_{i}=P_{1} L_{i}, \\
\beta=\alpha^{2}
\end{gathered}
$$

we establish the LMIs given by (27) in Theorem 1.

Thus, from (25) the LMI constraints (27) imply that $\widehat{X}_{1}$ exponentially converges to the unknown trajectory $X_{1}$ of system (10) which are identical to those of system (1). And, from system (24) and the fact that $\widehat{X}_{1}$ converges to $X_{1}, \widehat{X}_{2}$ exponentially converges to the unknown trajectory $X_{2}$ of system (10) which is identical to that of system (1).

\section{Application to a Heat Exchanger System}

The aim of this section consists in applying the above fuzzy observer design (12) with unmeasurable premise variables to a descriptor model of a heat exchanger pilot process.

4.1. Physical Model. The heat exchanger process considered is presented in Figure 1. The process is mainly built around a counterflow tubular heat exchanger. The warm water flows in a closed circuit, and the temperature in the hot water tank is fixed by an independently controlled electric heater. The cold water flows in an open circuit. The flows of either warm or cold water are controlled by two electropneumatic valves. $T_{1}, T_{3}$ are, respectively, the inlet temperatures of the warm and the cold water and $T_{2}, T_{4}$ are the correspondent outlet temperatures. The dynamics of actuators (electropneumatic valves) cannot be neglected. Indeed their time constants are equivalent to the residence time constants of the heat exchanger $(0.5 s-1 s)$. The correspondent state variables are the displacements and the velocities of the electropneumatic valves. The temperatures are assumed to be homogeneous in the tubular heat exchanger. Under the hypotheses that the circuit of the thermal exchanger is a closed system which contains a constant mass of water, the inertia of the fluid is negligible and the flow is turbulent.

The controlled variables of our problem are the temperatures $T_{2}$ and $T_{4}$, which are manipulated with the flows which are a function of electropneumatic valves current $I_{v w}$ and $I_{v c}$. The current on electropneumatic valve is an actual manipulated variable of the process. Furthermore, the heatexchanger is just one part of the plant. So, the actuators should also be modeled. The electropneumatic valve is a system that exhibits inherent second-order dynamics. For the heat-exchanger, we perform the energy balance for the characterization of the temperature. A descriptor model of the process takes the form

$$
\begin{gathered}
E \dot{x}=f(x)+g(x) u, \\
y=h(x),
\end{gathered}
$$

where $x=\left(x_{1}, \ldots, x_{8}\right)^{T}$ is the state vector, $u=\left(u_{1}, u_{2}\right)^{T}$ is the control vector, and $y=\left(x_{1}, x_{4}\right)^{T}=\left(T_{2}, T_{4}\right)^{T}$ is the output measurements. $x_{2}, x_{5}$ are, respectively, the displacement of the warm water valve and the cold water valve. $x_{3}, x_{6}$ are, respectively, the velocity of the warm water valve and the cold water valve. Finally, $x_{7}, x_{8}$ are, respectively, the acceleration of the warm water valve and the cold water valve:

$$
\begin{gathered}
f(x)=\left(\begin{array}{c}
e_{1} x_{2}-a_{1} x_{1} x_{2}-b_{1} x_{1}+b_{1} x_{4} \\
x_{3} \\
x_{7} \\
e_{2} x_{5}-a_{2} x_{4} x_{5}+b_{2} x_{1}-b_{2} x_{4} \\
x_{6} \\
x_{8} \\
-x_{7}-\omega_{0}^{2} x_{2}-2 \eta \omega_{0} x_{3} \\
-x_{8}-\omega_{0}^{2} x_{5}-2 \eta \omega_{0} x_{6}
\end{array}\right), \\
g(x)=\left(\begin{array}{cc}
0 & 0 \\
0 & 0 \\
0 & 0 \\
0 & 0 \\
0 & 0 \\
0 & 0 \\
k_{0} \omega_{0}^{2} & 0 \\
0 & k_{0} \omega_{0}^{2}
\end{array}\right)
\end{gathered}
$$




$$
\begin{aligned}
E= & \left(\begin{array}{llllllll}
1 & 0 & 0 & 0 & 0 & 0 & 0 & 0 \\
0 & 1 & 0 & 0 & 0 & 0 & 0 & 0 \\
0 & 0 & 1 & 0 & 0 & 0 & 0 & 0 \\
0 & 0 & 0 & 1 & 0 & 0 & 0 & 0 \\
0 & 0 & 0 & 0 & 1 & 0 & 0 & 0 \\
0 & 0 & 0 & 0 & 0 & 1 & 0 & 0 \\
0 & 0 & 0 & 0 & 0 & 0 & 0 & 0 \\
0 & 0 & 0 & 0 & 0 & 0 & 0 & 0
\end{array}\right), \\
& h(x)=\left(\begin{array}{l}
x_{1} \\
x_{4}
\end{array}\right) ;
\end{aligned}
$$

$a_{1}, a_{2}, b_{1}, b_{2}, e_{1}$, and $e_{2}$ are physical constants which derive from the energy balance transfer.

$k_{0}$ is the static gain of the valve, $\omega_{0}$ is the undamped natural frequency, and finally $\eta$ is the damping factor.

4.2. Takagi-Sugeno Descriptor Model. To express the model of the heat exchanger system as a Takagi-Sugeno model with the unmeasurable parameters (displacements of the valves $x_{2}$ and $x_{5}$ ) as decision variables, we use the procedure of fuzzy model construction given in [26]. For this purpose, we rewrite (40) in the following equivalent state space form:

$$
\begin{gathered}
E \dot{x}=A(x) x+B u, \\
y=C x,
\end{gathered}
$$

where

$$
B=g(x), \quad C=\left(\begin{array}{llllllll}
1 & 0 & 0 & 0 & 0 & 0 & 0 & 0 \\
0 & 0 & 0 & 1 & 0 & 0 & 0 & 0
\end{array}\right),
$$$$
A(x)=\left(\begin{array}{cccccccc}
-b_{1}-a_{1} x_{2} & e_{1} & 0 & b_{1} & 0 & 0 & 0 & 0 \\
0 & 0 & 1 & 0 & 0 & 0 & 0 & 0 \\
0 & 0 & 0 & 0 & 0 & 0 & 1 & 0 \\
b_{2} & 0 & 0 & -b_{2}-a_{2} x_{5} & e_{2} & 0 & 0 & 0 \\
0 & 0 & 0 & 0 & 0 & 1 & 0 & 0 \\
0 & 0 & 0 & 0 & 0 & 0 & 0 & 1 \\
0 & -w_{0}^{2} & -2 \eta w_{0} & 0 & 0 & 0 & -1 & 0 \\
0 & 0 & 0 & 0 & -w_{0}^{2} & -2 \eta w_{0} & 0 & -1
\end{array}\right) .
$$

Then, we consider the sector of the nonlinearities of the terms $\xi_{j} \in\left[\xi_{j \min }, \xi_{j \max }\right]$ of the matrix $A(x(t))$ with $j=1,2$ :

$$
\begin{aligned}
& \xi_{1}=-b_{1}-a_{1} x_{2}, \\
& \xi_{2}=-b_{2}-a_{2} x_{5} .
\end{aligned}
$$

Thus, we can transform the nonlinear terms under the following shape:

$$
\xi_{j}=M_{1 j} \xi_{j \max }+M_{2 j} \xi_{j \min } ; \quad j=\{1,2\},
$$

where

$$
\begin{aligned}
& M_{1 j}=\frac{\xi_{j}-\xi_{j \min }}{\xi_{j \max }-\xi_{j \min }}, \\
& M_{2 j}=\frac{\xi_{j \max }-\xi_{j}}{\xi_{j \max }-\xi_{j \min }} .
\end{aligned}
$$

Then, the global fuzzy model is inferred as

$$
\begin{gathered}
E \dot{x}=\sum_{i=1}^{4} h_{i}(x)\left(A_{i} x+B u\right), \\
y=C x,
\end{gathered}
$$

where

$$
\begin{aligned}
& h_{1}(x)=M_{21} M_{22} \text {, } \\
& h_{2}(x)=M_{21} M_{12} \text {, } \\
& h_{3}(x)=M_{11} M_{22} \text {, } \\
& h_{4}(x)=M_{11} M_{12} \text {, } \\
& A_{1}=\left(\begin{array}{cccccccc}
\xi_{1 \text { min }} & e_{1} & 0 & b_{1} & 0 & 0 & 0 & 0 \\
0 & 0 & 1 & 0 & 0 & 0 & 0 & 0 \\
0 & 0 & 0 & 0 & 0 & 0 & 1 & 0 \\
b_{2} & 0 & 0 & \xi_{2 \min } & e_{2} & 0 & 0 & 0 \\
0 & 0 & 0 & 0 & 0 & 1 & 0 & 0 \\
0 & 0 & 0 & 0 & 0 & 0 & 0 & 1 \\
0 & -w_{0}^{2} & -2 \eta w_{0} & 0 & 0 & 0 & -1 & 0 \\
0 & 0 & 0 & 0 & -w_{0}^{2} & -2 \eta w_{0} & 0 & -1
\end{array}\right), \\
& A_{2}=\left(\begin{array}{cccccccc}
\xi_{1 \min } & e_{1} & 0 & b_{1} & 0 & 0 & 0 & 0 \\
0 & 0 & 1 & 0 & 0 & 0 & 0 & 0 \\
0 & 0 & 0 & 0 & 0 & 0 & 1 & 0 \\
b_{2} & 0 & 0 & \xi_{2 \max } & e_{2} & 0 & 0 & 0 \\
0 & 0 & 0 & 0 & 0 & 1 & 0 & 0 \\
0 & 0 & 0 & 0 & 0 & 0 & 0 & 1 \\
0 & -w_{0}^{2} & -2 \eta w_{0} & 0 & 0 & 0 & -1 & 0 \\
0 & 0 & 0 & 0 & -w_{0}^{2} & -2 \eta w_{0} & 0 & -1
\end{array}\right), \\
& A_{3}=\left(\begin{array}{cccccccc}
\xi_{1 \max } & e_{1} & 0 & b_{1} & 0 & 0 & 0 & 0 \\
0 & 0 & 1 & 0 & 0 & 0 & 0 & 0 \\
0 & 0 & 0 & 0 & 0 & 0 & 1 & 0 \\
b_{2} & 0 & 0 & \xi_{2 \min } & e_{2} & 0 & 0 & 0 \\
0 & 0 & 0 & 0 & 0 & 1 & 0 & 0 \\
0 & 0 & 0 & 0 & 0 & 0 & 0 & 1 \\
0 & -w_{0}^{2} & -2 \eta w_{0} & 0 & 0 & 0 & -1 & 0 \\
0 & 0 & 0 & 0 & -w_{0}^{2} & -2 \eta w_{0} & 0 & -1
\end{array}\right), \\
& A_{4}=\left(\begin{array}{cccccccc}
\xi_{1 \max } & e_{1} & 0 & b_{1} & 0 & 0 & 0 & 0 \\
0 & 0 & 1 & 0 & 0 & 0 & 0 & 0 \\
0 & 0 & 0 & 0 & 0 & 0 & 1 & 0 \\
b_{2} & 0 & 0 & \xi_{2 \max } & e_{2} & 0 & 0 & 0 \\
0 & 0 & 0 & 0 & 0 & 1 & 0 & 0 \\
0 & 0 & 0 & 0 & 0 & 0 & 0 & 1 \\
0 & -w_{0}^{2} & -2 \eta w_{0} & 0 & 0 & 0 & -1 & 0 \\
0 & 0 & 0 & 0 & -w_{0}^{2} & -2 \eta w_{0} & 0 & -1
\end{array}\right) .
\end{aligned}
$$

4.3. Fuzzy Observer Design. Based on the on-line measurements of the temperature of the warm water $x_{1}$ and the temperature of the cold water $x_{4}$, we will show that the previous result (12) can be used to estimate the displacement, the velocity, and the acceleration of the warm water valves $x_{2}, x_{3}$, and $x_{7}$ and the displacement, the velocity, and the acceleration of the cold water valves $x_{5}, x_{6}$, and $x_{8}$.

Using Section 3, the construction of the fuzzy descriptor observer algorithm for heat exchanger system requires that the above system (47) takes the form (10). 
To do so, let

$$
\begin{aligned}
& X_{1}=\left[\begin{array}{llllll}
x_{1} & x_{2} & x_{3} & x_{4} & x_{5} & x_{6}
\end{array}\right]^{T}, \quad X_{2}=\left[\begin{array}{ll}
x_{7} & x_{8}
\end{array}\right]^{T}, \\
& E=\left(\begin{array}{ll}
I & 0 \\
0 & 0
\end{array}\right) \text { with } \operatorname{rank}(E)=6 \text {, } \\
& A_{i}=\left(\begin{array}{ll}
A_{11 i} & A_{12 i} \\
A_{21 i} & A_{22 i}
\end{array}\right) \\
& =\left(\begin{array}{ll}
A_{i}(1: 6,1: 6) & A_{i}(1: 6,7: 8) \\
A_{i}(7: 8,1: 6) & A_{i}(7: 8,7: 8)
\end{array}\right), \\
& \text { for } i=1,2,3,4 \text {, } \\
& B=\left(\begin{array}{l}
B_{1} \\
B_{2}
\end{array}\right)=\left(\begin{array}{l}
B(1: 6,1: 2) \\
B(7: 8,1: 2)
\end{array}\right), \\
& C=\left(C_{1} C_{2}\right)=(C(1: 2,1: 6) C(1: 2,7: 8)) \text {. }
\end{aligned}
$$

Notice that in this application $A_{22 i}=A_{i}(7: 8,7: 8)=$ $\left(\begin{array}{cc}-1 & 0 \\ 0 & -1\end{array}\right)$ are invertible. (1).

This shows that system (47) is a particular case of system

Consequently, from Theorem 1 a fuzzy observer for T-S descriptor system (47) permitting to estimate $x_{2}, x_{3}, x_{5}, x_{6}$, $x_{7}$, and $x_{8}$ takes the following form:

$$
\begin{aligned}
\dot{\hat{X}}_{1} & =\sum_{i=1}^{4} \bar{h}_{i}\left(\widehat{X}_{1}, u\right)\left(M_{i} \widehat{X}_{1}+N_{i} u-L_{i}(\widehat{y}-y)\right), \\
\widehat{X}_{2} & =\sum_{i=1}^{4} \bar{h}_{i}\left(\widehat{X}_{1}, u\right)\left(Q_{i} \widehat{X}_{1}+R_{i} u\right), \\
\widehat{y} & =\sum_{i=1}^{4} \bar{h}_{i}\left(\widehat{X}_{1}, u\right)\left(S_{i} \widehat{X}_{1}+G_{i} u\right),
\end{aligned}
$$

where $M_{i}, N_{i}, Q_{i}, R_{i}, S_{i}, G_{i}, \bar{h}_{i}$, and $L_{i}$ are given in the above equations (9), (11), and (29).

4.4. Simulation Results. In this section the purpose is to show by numerical simulations the good performances of the present study given in this paper. For all computer simulations results discussed in the sequel, we use the parameter values summarized in Table 1.

To simulate descriptor models (40) and (47), we use a Runge-Kutta method combined with the Newton-Raphson algorithm.

The initial conditions of the nonlinear system (40) and T-S model (47) are

$$
\begin{array}{cc}
x_{1}(0)=73^{\circ} \mathrm{C}, & x_{2}(0)=0 \mathrm{~m}, \\
x_{3}(0)=0 \mathrm{~m} / \mathrm{s}, & x_{4}(0)=18^{\circ} \mathrm{C}, \\
x_{5}(0)=0 \mathrm{~m}, & x_{6}(0)=0 \mathrm{~m} / \mathrm{s}, \\
x_{7}(0)=0.4406 \mathrm{~m} / \mathrm{s}^{2}, & x_{8}(0)=0.4406 \mathrm{~m} / \mathrm{s}^{2} .
\end{array}
$$

TABLE 1: List of parameters.

\begin{tabular}{lc}
\hline Parameters & Values \\
\hline$a_{1}$ & 552.5871 \\
$a_{2}$ & 92.0978 \\
$b_{1}$ & 0.2856 \\
$b_{2}$ & 0.0952 \\
$e_{1}$ & $4.1444 * 10^{4}$ \\
$e_{2}$ & $1.4736 * 10^{3}$ \\
$k_{0}$ & 0.93 \\
$w_{0}$ & 6.2832 \\
$\eta$ & 0.7 \\
$u_{1}$ & 0.012 \\
$u_{2}$ & 0.012 \\
\hline
\end{tabular}

First, we compare in Figure 2 the behavior of the continuous descriptor model (40) with its T-S model (47). For the considered T-S model, we can see that the T-S model represents exactly the nonlinear model.

In order to illustrate the performances of the T-S fuzzy observer (50), we solve the LMIs given in Theorem 1 . Then, note that in this practical case $C_{2}=0$; this implies that (see (9)) $S_{1}=\cdots=S_{q}=C_{1}$ and $G_{1}=\cdots=G_{q}=0$. Thus, the output of the system takes the following expression:

$$
y=C_{1} X_{1} .
$$

Now, the LMIs (27) given in Theorem 1 become

$$
\left(\begin{array}{ccc}
\mathscr{Z}_{1 j} & \Theta_{i j} & \Psi_{i j} \\
\Theta_{i j}^{T} & \mathscr{E}_{2 i} & P_{2} N_{i} \\
\Psi_{i j}^{T} & N_{i}^{T} P_{2} & -\beta I
\end{array}\right)<0 \quad \forall(i, j) \in\{1, \ldots, q\}^{2},
$$

where

$$
\begin{aligned}
\mathscr{Z}_{1 j} & =M_{j}^{T} P_{1}+P_{1} M_{j}-\mathscr{K}_{j} C_{1}-C_{1}^{T} \mathscr{K}_{j}^{T}+I, \\
\mathscr{Z}_{2 i} & =M_{i}^{T} P_{2}+P_{2} M_{i}, \\
\Theta_{i j} & =-P_{1}\left(M_{i}-M_{j}\right), \\
\Psi_{i j} & =-P_{1}\left(N_{i}-N_{j}\right) .
\end{aligned}
$$

Therefore, we solve the LMIs given in (53)-(54); we obtain the following observer gains $L_{i}, i=1,2,3,4$, and the minimal value of the attenuation level $\alpha$ :

$$
\begin{aligned}
& L_{1}=10^{7}\left(\begin{array}{cc}
2.9231 & 0.2860 \\
0.0000 & -0.0000 \\
-0.0000 & 0.0000 \\
0.2489 & 0.4752 \\
-0.0000 & 0.0000 \\
0.0000 & -0.0000
\end{array}\right), \\
& L_{2}=10^{7}\left(\begin{array}{cc}
2.9465 & 0.0162 \\
0.0000 & -0.0000 \\
-0.0000 & 0.0000 \\
0.2894 & 0.4921 \\
-0.0000 & 0.0000 \\
0.0000 & -0.0000
\end{array}\right),
\end{aligned}
$$



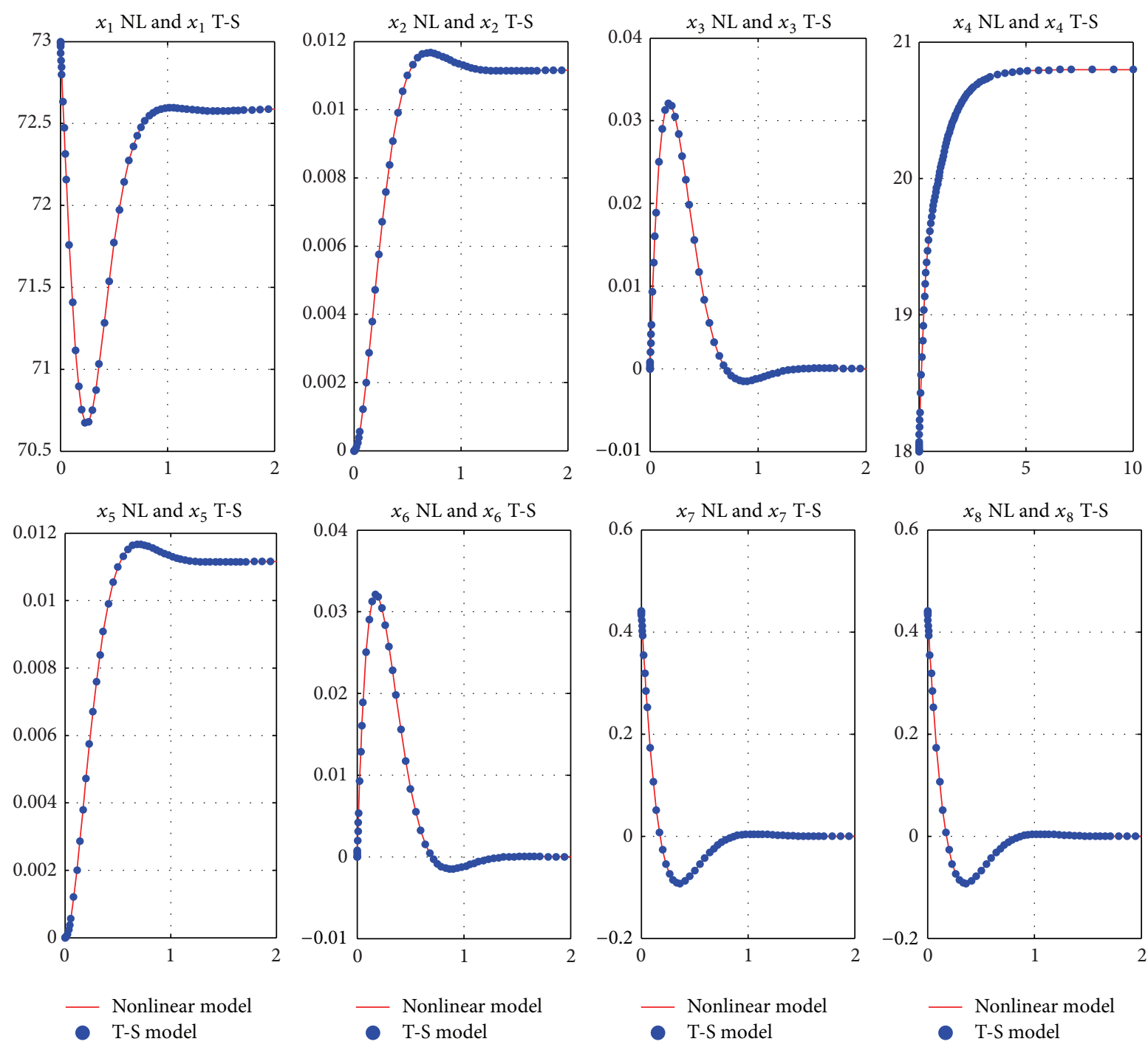

Figure 2

$$
\begin{gathered}
L_{3}=10^{8}\left(\begin{array}{cc}
0.5899 & -3.2133 \\
0.0000 & -0.0000 \\
0.0000 & 0.0000 \\
0.5469 & -0.2332 \\
0.0000 & 0.0000 \\
-0.0000 & -0.0000
\end{array}\right), \\
L_{4}=10^{7}\left(\begin{array}{cc}
3.4557 & -3.3143 \\
0.0000 & -0.0000 \\
-0.0000 & 0.0000 \\
0.8855 & 0.1733 \\
0.0000 & 0.0000 \\
-0.0000 & -0.0000
\end{array}\right), \\
\alpha=\sqrt{\beta}=4.4555 .
\end{gathered}
$$

$$
\begin{array}{cc}
\widehat{x}_{5}(0)=0.001 \mathrm{~m}, & \widehat{x}_{6}(0)=0.001 \mathrm{~m} / \mathrm{s}, \\
\widehat{x}_{7}(0)=0.3528 \mathrm{~m} / \mathrm{s}^{2}, & \widehat{x}_{8}(0)=0.3923 \mathrm{~m} / \mathrm{s}^{2} .
\end{array}
$$

Simulations results given in Figure 3 show the performances of the observer designed above with the parameters $L_{i}, i=$ $1,2,3,4$, where the dotted lines denote the state variables estimated by the fuzzy observer (50). This simulation shows that the estimation states converge to their corresponding state variables.

\section{Conclusion}

The initial conditions of the fuzzy observer (50) are

$$
\begin{array}{cc}
\widehat{x}_{1}(0)=73^{\circ} \mathrm{C}, & \widehat{x}_{2}(0)=0.002 \mathrm{~m}, \\
\widehat{x}_{3}(0)=0.001 \mathrm{~m} / \mathrm{s}, & \widehat{x}_{4}(0)=18^{\circ} \mathrm{C},
\end{array}
$$

In this paper, a new method to synthesize observer for continuous-time T-S descriptor model with unmeasurable premise variables was presented. The approach is based on the separation between dynamic and static relations. The convergence conditions are obtained by using Lyapunov theory and the $\mathscr{L}_{2}$ techniques. The existence of conditions 

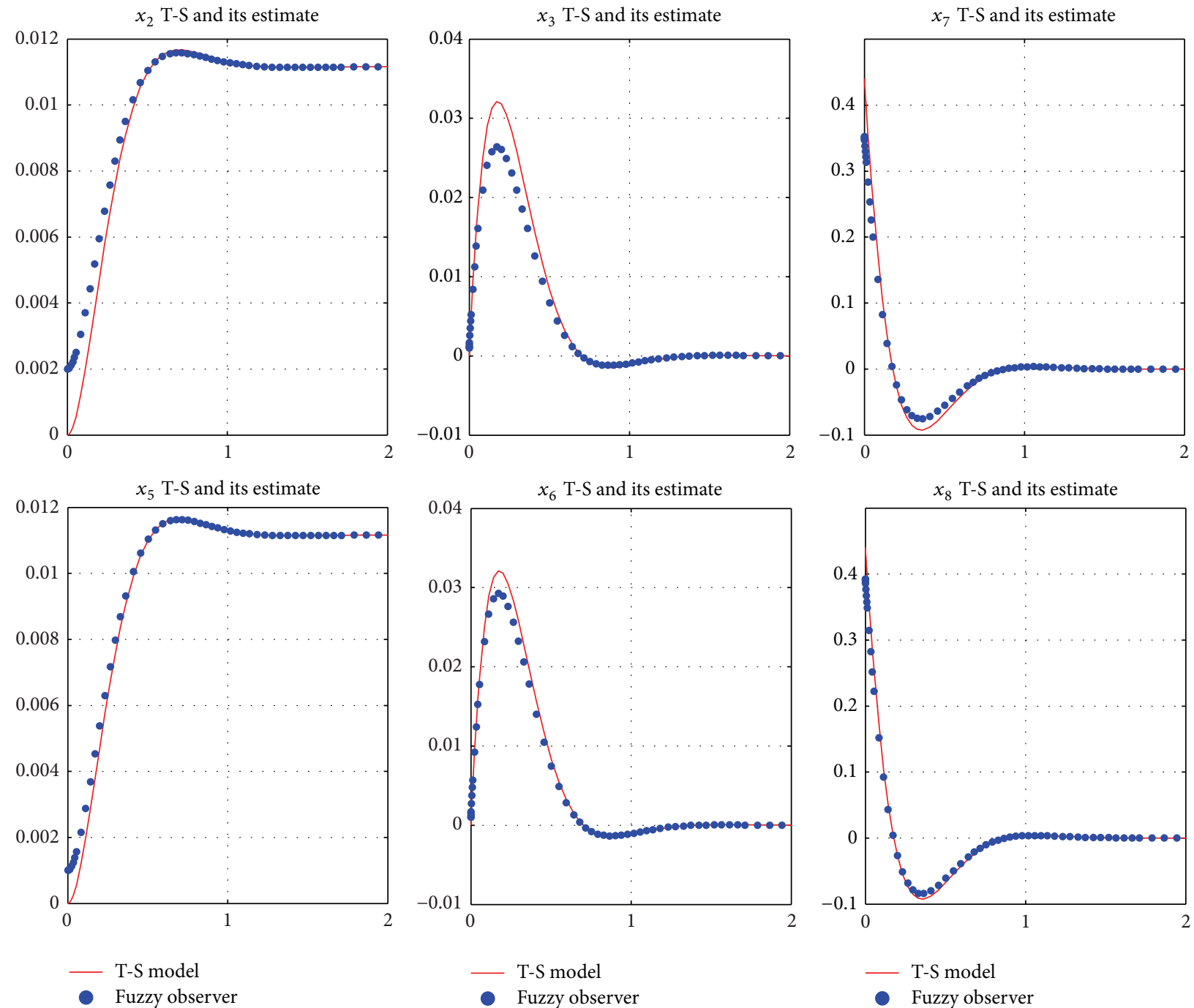

Figure 3

ensuring the convergence of the state estimation error is expressed in terms of LMIs. The proposed fuzzy observer is used for the on-line estimation of unknown state in a heat exchanger model. First, the Takagi-Sugeno fuzzy model is developed to represent the descriptor nonlinear model of the heat exchanger. Next, simulation results have been given and they demonstrated the good performances of the estimator.

\section{Conflict of Interests}

The authors declare that there is no conflict of interests regarding the publication of this paper.

\section{References}

[1] S. L. Campbell, Singular Systems of Differential Equations, Pitman, London, UK, 1980.

[2] F. L. Lewis, "A survey of linear singular systems," Circuits, Systems, and Signal Processing, vol. 5, no. 1, pp. 3-36, 1986.
[3] L. Dai, Singular Control Systems, Springer, Berlin, Germany, 1989.

[4] T. Takagi and M. Sugeno, "Fuzzy identification of systems and its applications to modeling and control," IEEE Transactions on Systems, Man and Cybernetics, vol. 15, no. 1, pp. 116-132, 1985.

[5] M. Chadli, D. Maquin, and J. Ragot, "Non quadratic stability analysis of Takagi Sugeno systems," in Proceedings of the IEEE Conference on Decision and Control (CDC '02), Las Vegas, Nev, USA, 2002.

[6] T. M. Guerra, A. Kruszewski, L. Vermeiren, and H. Tirmant, "Conditions of output stabilization for nonlinear models in the Takagi-Sugeno's form," Fuzzy Sets and Systems, vol. 157, no. 9, pp. 1248-1259, 2006.

[7] A. Kruszewski, R. Wang, and T. M. Guerra, "Nonquadratic stabilization conditions for a class of uncertain nonlinear discrete time TS fuzzy models: a new approach," IEEE Transactions on Automatic Control, vol. 53, no. 2, pp. 606-611, 2008.

[8] H. Zhang, Y. Shi, and J. Wang, "On energy-to-peak filtering for nonuniformly sampled nonlinear systems: a markovian jump system approach," IEEE Transactions on Fuzzy Systems, vol. 22, no. 1, pp. 212-222, 2014. 
[9] M. Liu, X. Liu, Y. Shi, and S. Wang, "T-S fuzzy-model-based $\mathrm{H}_{2}$ and $H_{\infty}$ filtering for networked control systems with twochannel Markovian random delays," Digital Signal Processing, vol. 27, no. 1, pp. 167-174, 2014.

[10] K. Tanaka and M. Sano, "On the concept of fuzzy regulators and fuzzy observers," in Proceedings of 3rd IEEE International Conference on Fuzzy Systems, vol. 2, pp. 767-772, June 1994.

[11] K. Tanaka and H. O. Wang, "Fuzzy regulators and fuzzy observers: a linear matrix inequality approach," in Proceedings of the 36th IEEE Conference on Decision and Control, vol. 2, pp. 1315-1320, San Diego, Calif, USA, December 1997.

[12] K. Tanaka, T. Ikeda, and H. O. Wang, "Fuzzy regulators and fuzzy observers: Relaxed stability conditions and LMI-based designs," IEEE Transactions on Fuzzy Systems, vol. 6, no. 2, pp. 250-265, 1998.

[13] T. A. Johansen, R. Shorten, and R. Murray-Smith, "On the interpretation and identification of dynamic Takagi-Sugeno fuzzy models," IEEE Transactions on Fuzzy Systems, vol. 8, no. 3, pp. 297-313, 2000.

[14] P. Bergsten and R. Palm, “Thau-Luenberger observers for TS fuzzy systems," in Proceedings of the 9th IEEE International Conference on Fuzzy Systems (FUZZ IEEE '00), vol. 2, pp. 671676, IEEE, San Antonio, Tex, USA, May 2000.

[15] K. Tanaka and H. O. Wan, Fuzzy Control Systems Design and Analysis: A Linear Matrix Inequality Approach, John Wiley \& Sons, 2001.

[16] P. Bergsten, R. Palm, and D. Driankov, "Fuzzy observers," in Proceedings of the IEEE International Fuzzy Systems Conference, Melbourne, Australia, 2001.

[17] P. Bergsten, R. Palm, and D. Driankov, "Observers for TakagiSugeno fuzzy systems," IEEE Transactions on Systems, Man, and Cybernetics B: Cybernetics, vol. 32, no. 1, pp. 114-121, 2002.

[18] D. Ichalal, B. Marx, J. Ragot, and D. Maquin, "Design of observers for Takagi-Sugeno systems with immeasurable premise variables: an $L_{2}$ approach," in Proceedings of the 17th World Congress, International Federation of Automatic Control (IFAC '08), Seoul, Korea, July 2008.

[19] D. Ichalal, B. Marx, J. Ragot, and D. Maquin, "State and unknown input estimation for nonlinear systems described by Takagi-Sugeno models with unmeasurable premise variables," in Proceedings of the 17th Mediterranean Conference on Control and Automation (MED '09), pp. 217-222, Thessaloniki, Greece, June 2009.

[20] A. M. Nagy, G. Mourot, B. Marx, G. Schutz, and J. Ragot, "Model structure simplification of a biological reactor," in Proceedings of the 15th IFAC Symposium on System Identification (SYSID '09), pp. 257-262, Saint-Malo, France, July 2009.

[21] T. Taniguchi, K. Tanaka, and H. O. Wang, "Fuzzy descriptor systems and nonlinear model following control," IEEE Transactions on Fuzzy Systems, vol. 8, no. 4, pp. 442-452, 2000.

[22] C. Lin, Q.-G. Wang, and T. H. Lee, "Stability and stabilization of a class of fuzzy time-delay descriptor systems," IEEE Transactions on Fuzzy Systems, vol. 14, no. 4, pp. 542-551, 2006.

[23] M. Essabre, J. Soulami, and E. Elyaagoubi, "Design of state observer for a class of non linear singular systems described by Takagi-Sugeno model," Contemporary Engineering Sciences, vol. 6, no. 1-4, pp. 99-109, 2013.

[24] H. Hamdi, M. Rodrigues, C. Mechmech, and N. Benhadj Braiek, "Observer based fault tolerant control for Takagi-Sugeno nonlinear descriptor systems," in Proceedings of the International Conference on Control, Engineering \& Information Technology (CEIT '13), vol. 1, pp. 52-57, 2013.
[25] M. Essabre, J. Soulami, A. El Assoudi, E. H. El Yaagoubi, and E. M. El Bouatmani, "Fuzzy observer design for a class of takagisugeno descriptor systems," Contemporary Engineering Sciences, vol. 7, no. 1-4, pp. 149-164, 2014.

[26] T. Taniguchi, K. Tanaka, H. Ohtake, and H. O. Wang, "Model construction, rule reduction, and robust compensation for generalized form of Takagi-Sugeno fuzzy systems," IEEE Transactions on Fuzzy Systems, vol. 9, no. 4, pp. 525-538, 2001. 

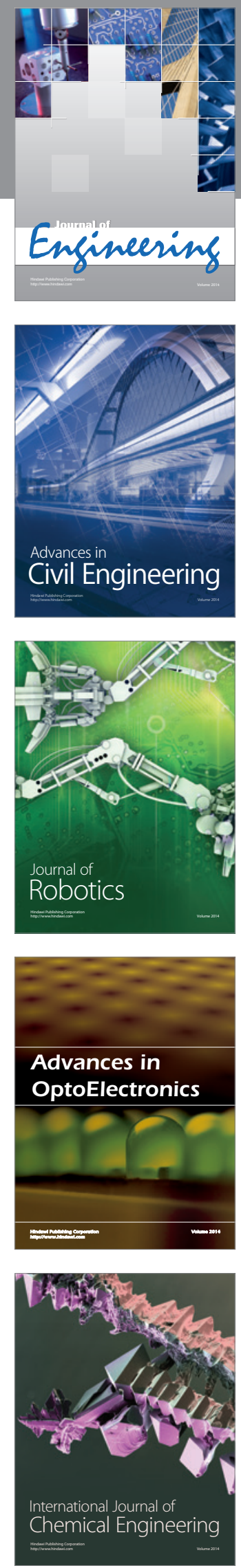

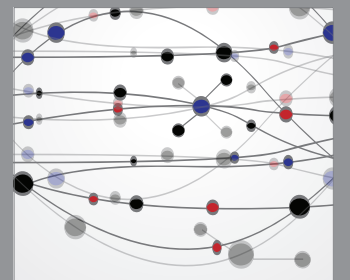

The Scientific World Journal
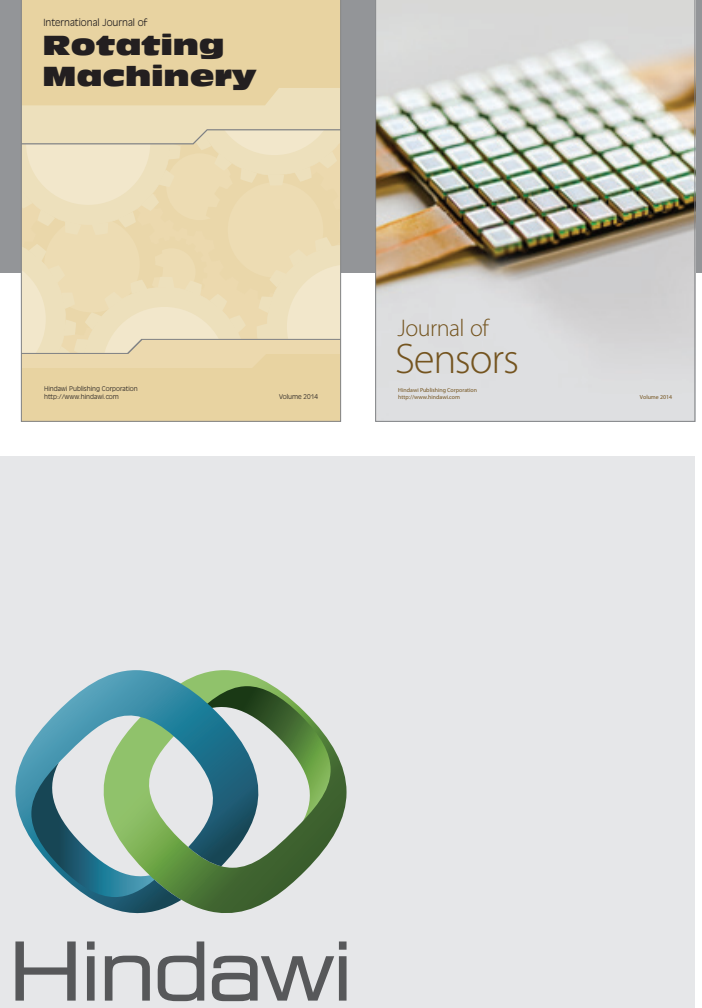

Submit your manuscripts at http://www.hindawi.com
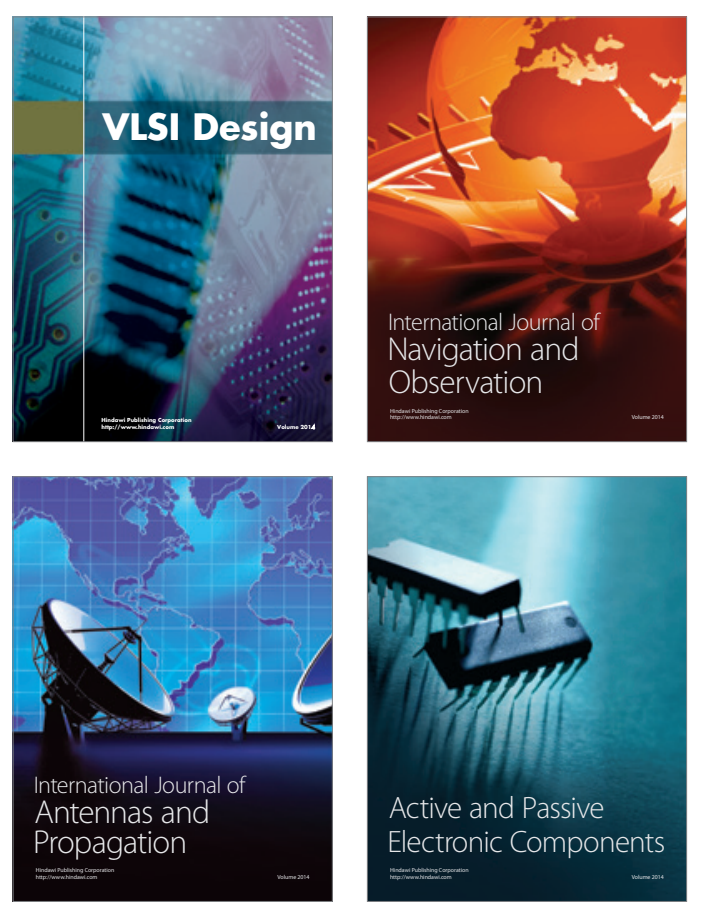
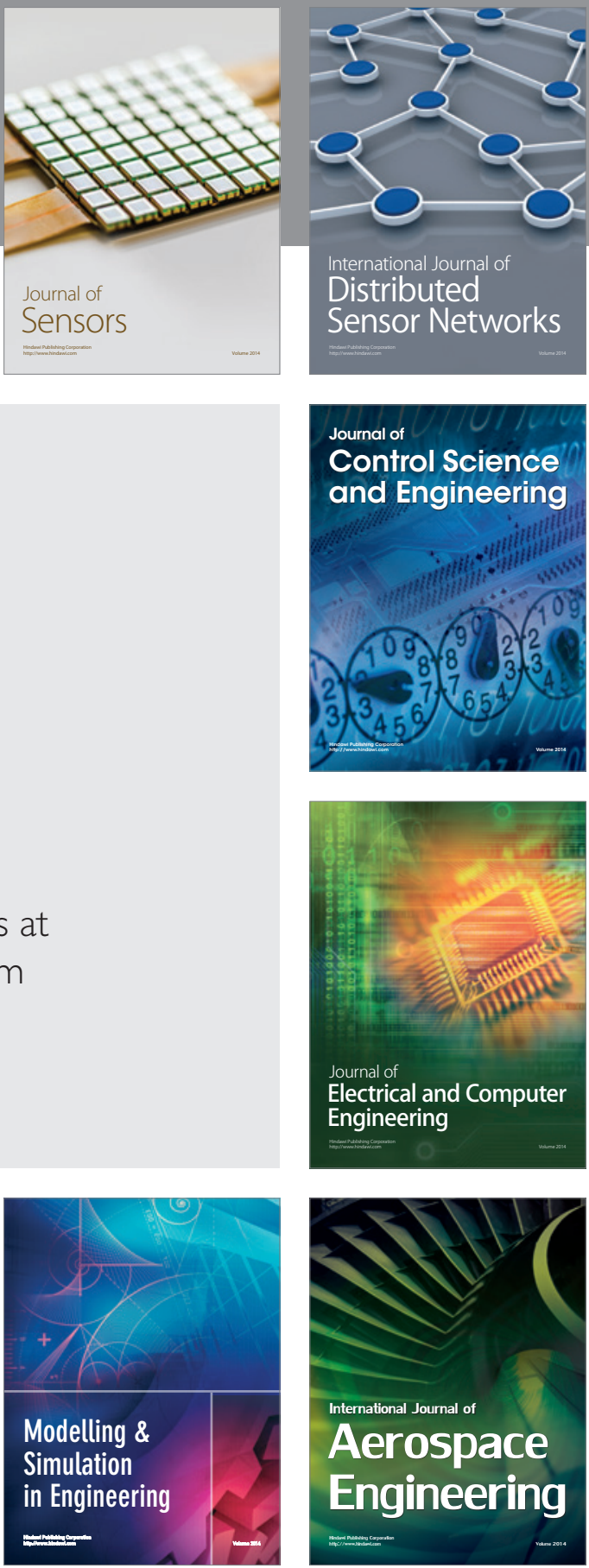

Journal of

Control Science

and Engineering
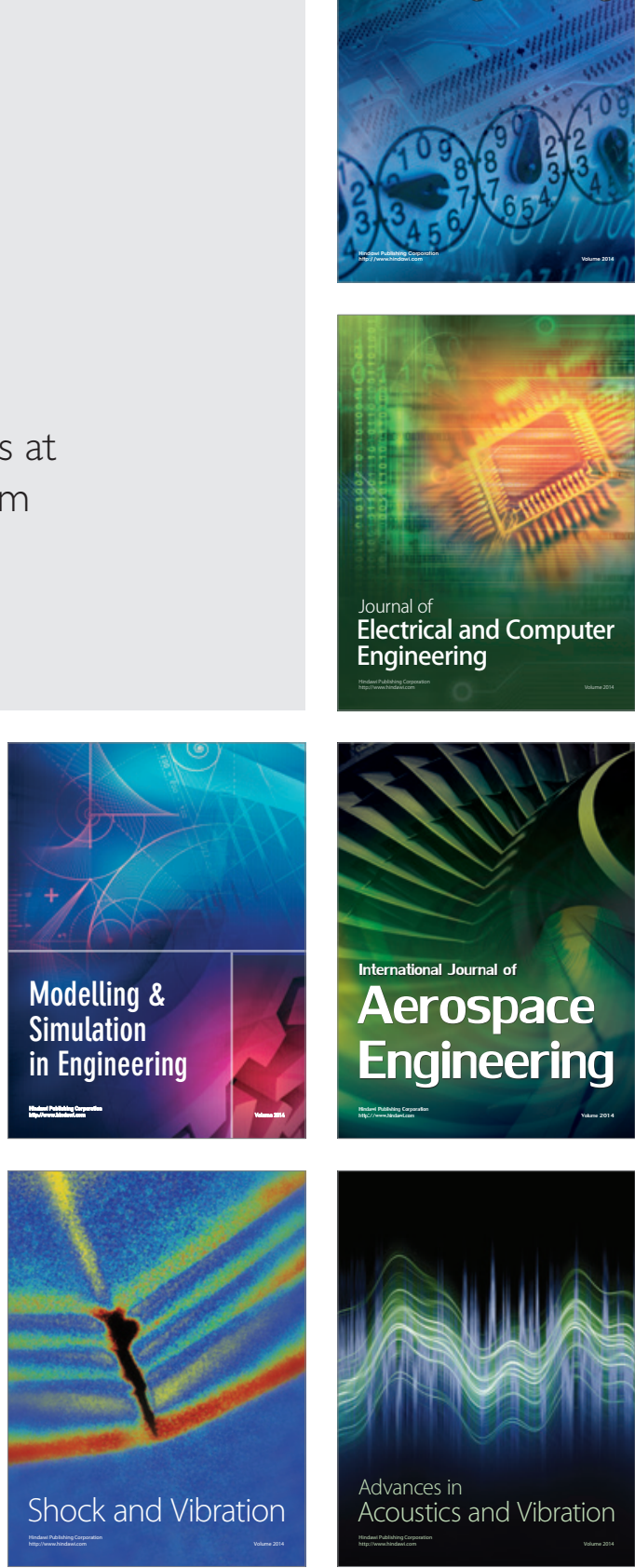